\title{
A NONLINEAR STEADY STATE TEMPERATURE PROBLEM
}

\section{W. ROBERT MANN AND JACOB F. BLACKBURN ${ }^{1}$}

This paper is devoted to a study of the following nonlinear boundary value problem.

$$
\begin{array}{rlrl}
U_{x x}+U_{y y} & =0 & \text { for } 0<x<\pi \text { and } 0<\nu<\infty, \\
U(0, y) & =U(\pi, y)=0 & \text { for } 0 \leqq y<\infty, \\
U_{y}(x, 0) & =-G[U(x, 0)] & \text { for } 0<x<\pi, \\
|U(x, y)| & <M \text { for } 0 \leqq x \leqq \pi \text { and } 0 \leqq y<\infty \text { for some } M .
\end{array}
$$

It is understood of course that $U(x, y)$ and $U_{y}(x, y)$ are continuous on the closed semi-infinite strip, and we shall also assume that $U_{x x}$ and $U_{y y}$ are continuous bounded functions of $x$ for each $y>0$. Concerning $G$, the following three assumptions are made:

A. $G[U]$ is continuous.

B. $G[U]$ is a decreasing function of $U$.

C. $G[1]=0$.

We shall call every function having these three properties a gradient function.

The above system is easily recognized as the mathematical model corresponding to the steady state temperature distribution in a semiinfinite strip of width $\pi$ with the short side exposed to a gas maintained constantly at unit temperature. Boundary condition (1b) generalizes Newton's Law of cooling which in the corresponding linear problem would be

$$
k U_{y}(x, 0)=-l \cdot[1-U(x, 0)]
$$

where $k$ and $l$ are familiar physical parameters ordinarily assumed to be constant. At high temperatures, where most of the heat transfer is by radiation, the well-known "fourth power law" implies that the factor $l$ in (2) varies considerably with $U(x, 0)$. For this reason we have replaced (2) by (1b) which, together with the three hypotheses $\mathrm{A}, \mathrm{B}$, and $\mathrm{C}$ on the function $G[U]$, is the most general physically significant boundary condition for heat transfer between solids and gases. $U_{y}(x, 0)$ is, except for a constant factor, the rate of heat flow

Presented to the Society, November 28, 1953; received by the editors April 29, 1954.

1 The junior author wishes to acknowledge the benefit he has received from several illuminating discussions on the subject of integral equations with $\mathrm{Mr}$. Adolf Nussbaum of the Institute for Advanced Study. 
from the gas to the solid, so (1b) in conjunction with hypothesis $B$ simply says that this rate is a decreasing function of the surface temperature of the solid. Hypothesis $\mathrm{C}$ is a consequence of the fact that when the surface of the solid is at the temperature of the gas the net rate of heat gain by the solid from the gas is zero.

The purpose of this paper is to prove that the problem stated has a unique solution which is bounded between 0 and 1 , and to point out a method of successive approximations which, under certain conditions, converges to $U(x, 0)$ for all $x$ on $[0, \pi]$.

The first step is to reduce the above problem to a nonlinear integral equation by application of a finite Fourier transform. Assuming, to begin with, that a solution $U(x, y)$ does exist, the statement of the problem implies that it has a finite Fourier sine transform with respect to $x$, say $u(n, y)$, which satisfies the following conditions:

$$
\begin{aligned}
& u_{y y}(n, y)-n^{2} u(n, y)=0, \\
& u_{y}(n, 0)=S\{-G[U(x, 0)]\}=-g(n), \\
& |u(n, y)|<M \pi .
\end{aligned}
$$

The solution of this transformed boundary value problem is easily seen to be

$$
u(n, y)=g(n) \cdot \frac{e^{-n y}}{n} .
$$

In order to invert $u(n, y)$ we use the convolution theorem [1]

$$
S^{-1}\left\{f_{s}(n) h_{c}(n)\right\}=\frac{1}{2} F_{1}(x)^{*} H_{2}(x)
$$

where $F$ and $H$ are two functions defined on $0<x<\pi ; F_{1}(x)$ denotes the odd periodic extension of $F(x)$ of period $2 \pi ; H_{2}(x)$ is the even extension of $H(x)$;

$$
\begin{aligned}
& f_{s}(n)=\int_{0}^{\pi} F_{1}(x) \sin n x d x, \quad n=1,2,3, \cdots, \\
& h_{c}(n)=\int_{0}^{\pi} H_{2}(x) \cos n x d x, \quad n=0,1,2, \cdots .
\end{aligned}
$$

From this we see that in order to invert $u(n, y)$ we must find out what function has $e^{-n y} / n$ for its finite cosine transform. This can easily be done by starting with the fact that

$$
\sum_{n=1}^{\infty} \frac{u^{n}}{n}=-\log (1-u)
$$


in the unit circle and on the circumference except at $u=1$. Substituting $u=e^{i z}$ and then equating real parts in the resulting equation gives

$$
\sum_{n=1}^{\infty} \frac{1}{n} e^{-n y} \cos n x=\frac{1}{2} \log \left(1-2 e^{-y} \cos x+e^{-2 y}\right) .
$$

This series converges uniformly not only for $y>0$ but also along the $x$-axis when $x \neq \equiv 0 \bmod 2 \pi$. Hence it is the Fourier series of its sum. From (5) it now follows that

$$
\begin{aligned}
U(x, y) & =S^{-1}\left\{g(n) \cdot \frac{e^{-n y}}{n}\right\} \\
& =-\frac{1}{2 \pi} \int_{-\pi}^{\pi} \log \left[1-2 e^{-y} \cos (x-\lambda)+e^{-2 y}\right] G_{1}[U(\lambda, 0)] d \lambda .
\end{aligned}
$$

This is easily seen to be the same as

(6) $U(x, y)=\frac{1}{2 \pi} \int_{0}^{\pi} \log \left[\frac{1-2 e^{-y} \cos (x+\lambda)+e^{-2 y}}{1-2 e^{-y} \cos (x-\lambda)+e^{-2 y}}\right] G[U(\lambda, 0)] d \lambda$.

In other words, if there exists a function $U(x, y)$ satisfying the original problem, it must be expressible in terms of $U(x, 0)$ through formula (6). From (6) and the continuity hypothesis on $U$ we get

$$
U(x, 0)=\frac{1}{2 \pi} \int_{0}^{\pi} \log \left[\frac{1-\cos (x+\lambda)}{1-\cos (x-\lambda)}\right] G[U(\lambda, 0)] d \lambda .
$$

We shall now show that, for every gradient function $G[U]$, equation $\left(7^{\prime}\right)$ has a unique bounded solution, and that this solution will always yield, through (6), a unique solution of our original boundary value problem.

Let $U(x, 0)=f(x)$ and

$$
K(x, \lambda)=\frac{1}{2 \pi} \log \left[\frac{1-\cos (x+\lambda)}{1-\cos (x-\lambda)}\right] .
$$

Then equation $\left(7^{\prime}\right)$ becomes

$$
f(x)=\int_{0}^{\pi} K(x, \lambda) G[f(\lambda)] d \lambda .
$$

Now evaluating the integral

$$
\frac{1}{2 \pi} \int_{0}^{\pi} \log \left[\frac{1-e^{i(x+\lambda)}}{1-e^{i(x-\lambda)}}\right] \frac{e^{i n \lambda}-e^{-i n \lambda}}{2 i} d \lambda
$$


by expanding the integrand in power series we get that

$$
\sin n x=n \int_{0}^{\pi} K(x, \lambda) \sin n \lambda d \lambda .
$$

In other words, the eigen-numbers of the kernel are the natural numbers and $\{\sin n x\}$ are the eigen-functions. (That these are the only eigen-functions follows from the fact that they form a complete system on $[0, \pi]$.) Since all the eigen-numbers are positive, it follows that the symmetric kernel $K(x, \lambda)$ is positive definite. $K(x, \lambda)$ has a singularity at $x=\lambda$ but it is nevertheless easily seen to be square integrable, and continuous in the mean, i.e.

$$
\Delta^{2}\left(x_{1}, x_{2}\right)=\int_{0}^{\pi}\left[K\left(x_{1}, y\right)-K\left(x_{2}, y\right)\right]^{2} d y \rightarrow 0 \text { as } x_{2} \rightarrow x_{1} .
$$

Hence the bilinear expansion is valid,

$$
K(x, \lambda)=\frac{2}{\pi} \sum_{1}^{\infty} \frac{\sin n x \sin n \lambda}{n},
$$

and also [2]

$$
K_{2}(x, y)=\int_{0}^{\pi} K(x, \lambda) K(\lambda, y) d \lambda
$$

$$
=\sum_{n=1}^{\infty} \frac{\sin n x \sin n y}{n^{2}}=\left\{\begin{array}{l}
\frac{x(\pi-y)}{\pi} \text { for } 0 \leqq x \leqq y, \\
\frac{y(\pi-x)}{\pi} \text { for } y \leqq x \leqq \pi .
\end{array}\right.
$$

From the Schwartz inequality and the continuity in the mean of $K$, the following theorem is easily proved.

THEOREM 1. Every bounded solution of (7) is continuous.

To prove the existence of a bounded solution of (7), we introduce the following functional transformation

$$
T[f](x)=\int_{0}^{\pi} \bar{G}[f(\lambda)] K(x, \lambda) d \lambda
$$

where

$$
\bar{G}[f]=\left\{\begin{array}{cl}
G[f] & \text { when } f \leqq 1 \\
0 & \text { when } f>1
\end{array}\right.
$$


Since $G[1]=0, \bar{G}$ is continuous. Consider the sequence of functions defined recursively as follows

$$
f_{n+1}(x)=T\left[f_{n}\right](x), \quad \text { where } f_{0}(x) \equiv 0 .
$$

This sequence is easily pictured if we make the following simple observations

$f_{n}(0)=f_{n}(\pi)=0, n=0,1,2, \cdots$.

$f_{1}(x)$ is concave down with a maximum value at $x=\pi / 2$.

$f_{n}(x) \geqq 0$ for all $n$ and for all $x$ on $[0, \pi]$.

$$
\begin{array}{rrrr}
u(x) & \geqq v(x) \quad \text { for } 0 \leqq x \leqq \pi \rightarrow T[u] x \leqq T[v](x), \\
f_{0}(x) & <f_{2}(x)<\cdots<f_{2 k}(x)<\cdots & \text { for } 0<x<\pi, \\
f_{1}(x) & >f_{3}(x)>\cdots>f_{2 k+1}(x)>\cdots & \text { for } 0<x<\pi, \\
f_{2 k+1}(x) & >f_{2 m}(x) & \text { for all } k \text { and } m & \text { when } 0<x<\pi .
\end{array}
$$

The sequence $\left\{f_{2 k}(x)\right\}$ converges from below to some function $F_{2}(x)$ and $\left\{f_{2 k+1}(x)\right\}$ converges from above to $F_{1}(x)$ where $F_{1}(x) \geqq F_{2}(x)$. If $F_{1}(x) \equiv F_{2}(x)$, then this function is a fixpoint of $(10)$ and can be shown to be a solution of (7). This will necessarily be the case if $T$ is a uniformly distance decreasing transformation, e.g. if $T$ satisfies a Lipschitz condition with constant less than 1 . Under this hypothesis the successive approximations defined by (10) converge in the large and an upper bound for the absolute value of the error in $f_{n}(x)$ is $\left|f_{n}(x)-f_{n-1}(x)\right|$, as is apparent from (13), (14), and (15).

But regardless of whether the sequence $\left\{f_{n}(x)\right\}$ converges, we can prove the existence of a solution of (7) by a simple application of the Schauder fixpoint theorem [3]. To this end let us consider the Banach space $B$ of continuous functions on $[0, \pi]$ with norm $\|f(x)\|$ $=\max |f(x)|$. By the set $A$ we shall mean those functions having the following properties:

(i) $f(x)$ belongs to $B$;

(ii) $0 \leqq f(x) \leqq f_{1}(x)$;

(iii) $\left|f\left(x_{1}\right)-f\left(x_{2}\right)\right| \leqq \pi^{1 / 2} G_{0} \Delta\left(x_{1}, x_{2}\right)$,

where $\Delta\left(x_{1}, x_{2}\right)$ is defined in (8).

Since the kernel $K$ is continuous in the mean, the functions comprising $A$ are equicontinuous, and since they are also equibounded it follows that $A$ is compact.

To show that $A$ is convex, consider $h(x)=\alpha f(x)+(1-\alpha) g(x)$ where $0 \leqq \alpha \leqq 1$ and both $f(x)$ and $g(x)$ belong to $A$. Obviously $h(x)$ is continuous and hence belongs to $B$. Moreover,

$$
0 \leqq h(x) \leqq \alpha f_{1}(x)+(1-\alpha) f_{1}(x)=f_{1}(x),
$$


and

$$
\begin{aligned}
\left|h\left(x_{1}\right)-h\left(x_{2}\right)\right| & \leqq \alpha\left|f\left(x_{1}\right)-f\left(x_{2}\right)\right|+(1-\alpha)\left|g\left(x_{1}\right)-g\left(x_{2}\right)\right| \\
& \leqq \alpha \pi^{1 / 2} G_{0} \Delta\left(x_{1}, x_{2}\right)+(1-\alpha) \pi^{1 / 2} G_{0} \Delta\left(x_{1}, x_{2}\right) \\
& =\pi^{1 / 2} G_{0} \Delta\left(x_{1}, x_{2}\right),
\end{aligned}
$$

so $h(x)$ belongs to $A$ and $A$ is convex.

We must now show that $T$ carries $A$ into itself continuously. It is obvious from (12) that $f_{0}(x) \leqq T[f](x) \leqq f_{1}(x)$ when $f(x)$ belongs to $A$. Consider now

$$
T[f]\left(x_{1}\right)-T[f]\left(x_{2}\right)=\int_{0}^{\pi} G[f(\lambda)]\left[K\left(x_{1}, \lambda\right)-K\left(x_{2}, \lambda\right)\right] d \lambda .
$$

By the Schwartz inequality we have

$$
\begin{aligned}
& \left|T[f]\left(x_{1}\right)-T[f]\left(x_{2}\right)\right|^{2} \\
& \quad \leqq \int_{0}^{\pi} G^{2}[f(\lambda)] d \lambda \int_{0}^{\pi}\left[K\left(x_{1}, \lambda\right)-K\left(x_{2}, \lambda\right)\right]^{2} d \lambda \leqq \pi G_{0}^{2} \Delta^{2}\left(x_{1}, x_{2}\right) .
\end{aligned}
$$

Therefore $T$ carries $A$ into itself. To see that $T$ is continuous, let $f$ and $g$ be any two elements of $A$.

$$
\begin{aligned}
\|T[f]-T[g]\| & =\max _{0 \leqq x \leqq \pi}\left|\int_{0}^{\pi}\{\bar{G}[f(\lambda)]-\bar{G}[g(\lambda)]\} K(x, \lambda) d \lambda\right| \\
& \leqq \max _{0 \leqq x \leqq \pi} \int_{0}^{\pi}\{\bar{G}[f(\lambda)]-\bar{G}[g(\lambda)]\}^{2} d \lambda \int_{0}^{\pi} K^{2}(x, \lambda) d \lambda .
\end{aligned}
$$

But by (9),

$$
\int_{0}^{\pi} K^{2}(x, \lambda) d \lambda=K_{2}(x, x) \leqq M
$$

Therefore

$$
\|T[f]-T[g]\| \leqq M \int_{0}^{\pi}\{\bar{G}[f(\lambda)]-\bar{G}[g(\lambda)]\}^{2} d \lambda
$$

and the continuity of $T$ follows from that of $\bar{G}$.

We have now proved that $A$ is a convex compact set in a Banach space and that $T$ carries $A$ into itself continuously. The Schauder fixpoint theorem yields immediately the following result.

THEOREM 2. There exists at least one continuous function, $f(x)$, on $[0, \pi]$ such that $0 \leqq f(x) \leqq T[0](x)$ and such that $f(x)=T[f](x)$. 
To infer the existence of a solution of (7) we need the following

THEOREM 3. If $f(x)$ is a continuous fixpoint of $T$, then $f(x) \leqq 1$ for $0 \leqq x \leqq \pi$.

Proof. Let $E$ be the set of points on which $f(x)>1$. Then $\bar{G}[f(x)]=0$ on $E$. Let $\tilde{E}$ be the complement of $E$ with respect to $[0, \pi]$. $E$ is an open set and at every point of $E, f(x)$ has a derivative given by

$$
f^{\prime}(x)=\int_{\widetilde{E}} K_{x}(x, \lambda) \bar{G}[f(\lambda)] d \lambda=\frac{1}{\pi} \int_{\widetilde{E}} \frac{\sin \lambda}{\cos x-\cos \lambda} \bar{G}[f(\lambda)] d \lambda
$$

and a second derivative given by

$$
f^{\prime \prime}(x)=\frac{1}{\pi} \int_{\widetilde{E}} \frac{\sin \lambda \sin x}{(\cos x-\cos \lambda)^{2}} \bar{G}[f(\lambda)] d \lambda .
$$

Since both factors of the integrand are non-negative it follows that $f^{\prime \prime}(x) \geqq 0$ on $E$ which means that $f(x)$ is concave upward at every point of $E$. This is a contradiction unless $E$ is empty. Therefore $f(x)$ $\leqq 1$ for $0 \leqq x \leqq \pi$.

THEorem 4. Equation (7) has a continuous solution, $f(x)$, on $[0, \pi]$ and $0 \leqq f(x) \leqq 1$.

This follows from Theorems 2 and 3 , since $G[f]=\bar{G}[f]$ when $f \leqq 1$.

Theorem 5. Equation (7) does not have more than one bounded solution on $[0, \pi]$.

Proof. Assume that $f_{1}(x)$ and $f_{2}(x)$ are both solutions. Then if we set $f_{1}(x)-f_{2}(x)=h(x)$ and $G\left[f_{1}(x)\right]-G\left[f_{2}(x)\right]=H(x)$ it follows from (7) that

$$
h(x)=\int_{0}^{\pi} K(x, \lambda) H(\lambda) d \lambda
$$

and

$$
\int_{0}^{\pi} h(x) H(x) d x=\int_{0}^{\pi} \int_{0}^{\pi} K(x, \lambda) H(\lambda) H(x) d \lambda d x .
$$

Since $G$ is a decreasing function, $h(x) H(x) \leqq 0$ and hence the lefthand side of the above equation is not positive. But since $K(x, \lambda)$ is positive definite, the right-hand side is non-negative and can be zero if and only if $H(x) \equiv 0$. This implies that $f_{1}(x) \equiv f_{2}(x)$.

Summarizing the results so far, we can say that a necessary condition for $U(x, y)$ to be a solution of the original boundary value prob- 
lem is that $U(x, y)$ be related to $U(x, 0)$ through $(6)$ where $U(x, 0)$ is a bounded solution of (7). For every gradient function $G$, equation (7) has a unique bounded solution, and this solution is continuous, positive, and nowhere greater than 1 . From this and (6) it is easily seen that $0 \leqq U(x, y) \leqq 1$ for $0 \leqq x \leqq \pi$ and $y \geqq 0$.

Having shown that every solution of the original boundary value problem is a bounded solution of (7) when $y=0$, the question arises as to whether, for all gradient functions $G$, the corresponding unique bounded solution of (7) will yield through (6) a solution of the original problem. This question can be answered in the affirmative by a somewhat lengthy process involving the standard techniques of treating improper integrals and interchanging order in multiple limit processes. Since there is no novelty involved we shall omit these details and merely state that the boundary value problem is equivalent to that of finding bounded solutions of (7).

\section{REFERENCES}

1. R. V. Churchill, Modern operational mathematics in engineering, New York and London, McGraw-Hill, 1944.

2. G. Hamel, Integralgleichungen, Berlin, Springer, 1937.

3. J. Schauder, Der Fixpunktsatz in Funktionalraeumen, Studia Mathematica vol. 2 (1930) pp. 171-180.

University of North Carolina and InSTITUTE for Advanced StUdy 